\title{
Quantitative Proteomic Analysis reveals similarities between Huntington's Disease (HD) and Huntington's disease-like 2 (HDL2) human brains
}

\author{
Tamara Ratovitski, ${ }^{1 *}$ Raghothama Chaerkady, ${ }^{2}$ Kai Kammers, ${ }^{3}$ Jacqueline C. Stewart, ${ }^{1}$ \\ Anialak Zavala, ${ }^{1}$ Olga Pletnikova, ${ }^{4}$ Juan C. Troncoso,${ }^{4}$ Dobrila D. Rudnicki, ${ }^{1}$ Russell L. \\ Margolis, ${ }^{1,5}$ Robert N. Cole ${ }^{2}$ and Christopher A. Ross. ${ }^{1,5,6 *}$
}

${ }^{1}$ Division of Neurobiology, Department of Psychiatry and Behavioral Sciences, Johns Hopkins University School of Medicine, 600 North Wolfe Street, CMSC 8-121, Baltimore, MD 21287

${ }^{2}$ Mass Spectrometry and Proteomics Facility, Department of Biological Chemistry, Johns Hopkins University School of Medicine, 733 N. Broadway Street, Suite 371 BRB Baltimore, MD 21205

${ }^{3}$ Department of Biostatistics, Johns Hopkins Bloomberg School of Public Health, Baltimore, MD 21205

${ }^{4}$ Department of Pathology, Johns Hopkins University School of Medicine, Baltimore, MD 21287

${ }^{5}$ Department of Neurology and Program in Cellular and Molecular Medicine, Johns Hopkins University School of Medicine, Baltimore, MD 21287

${ }^{6}$ Departments of Pharmacology and Neuroscience, Johns Hopkins University School of Medicine, Baltimore, MD 21287

*Address correspondence to: Christopher A. Ross (caross@jhu.edu) or Tamara Ratovitski (tratovi1@jhmi.edu), CMSC 8-121, 600 North Wolfe Street, Baltimore, MD 21287. Fax: 410614-0013 
Table of Content:

Supplementary figure legends

Supplementary figures S1-S5

Supplementary tables legends

IPA networks and pathways shapes key 


\section{Supplementary figures legends}

Figure S1 Mass-spectrometry-based QC for non-specific post-mortem autolysis. To rule out that any observed differences in protein abundance were due to differences in postmortem protein autolysis in different samples, the relative amount of semi-tryptic peptides of $\beta$-tubulin in HD brains versus control brain were compared within each of 3 iTRAQ experiments. Blue circles show $\log 2$ ratio distributions for reporter ions of semi-tryptic peptides used in calculating $\beta$-tubulin protein ratios for each HD sample compared to control for the three iTRAQ experiments. Blue box and whiskers plot the 5, 25, 75 and 95\% quantile distributions of the reporter ions from these semi-tryptic peptides. Red circles represent the distribution report ion ratios from tubulin peptides not used to calculate $\log 2$ ratios. These peptides were either complete tryptic peptides, peptide sequences not unique to $\beta$-tubulin or redundant ratios due to multiple search results from the same peptide fragmentation (MS2 or sequencing) spectrum. A $\log 2$ fold change of zero indicates no significant difference in postmortem autolysis between the samples

Figure S2. Preparation of brain samples for iTRAQ analysis. Motor cortex tissues from indicated HD and control cases were homogenized and centrifugated, and proteins were precipitated using 2-D Clean-up kit. Aliquots of the prepared material (50 $\mu \mathrm{g}$ of total protein) were fractionated on NuPAGE 4-12\% Bis-Tris polyacrylamide gels and stained with Coomassie protein stain.

Figure S3. Differentially abundant proteins related to Huntington's Disease. IPA "Diseases and Functions" analysis mapped 121 out of 1211 proteins, significantly changed in abundances in HD brains versus control, as related to Huntington's Disease. Proteins increased in abundance 
are in red, decreased-are in green. Only proteins with significant change in expression between HD and control $(\mathrm{q}<0.05)$ were included in analysis.

Figure S4. IPA analysis of top canonical pathways altered in HDL2. Top cellular pathways (as determined by IPA) most significantly enriched within the proteins that changed in abundances (both up and down) in HDL2 brain versus control (- $\log _{10}$ p-values are calculated by IPA). Only proteins significantly changed $(\mathrm{q}<0.05)$ were included in the analysis Figure S5. Comparative functional analysis (using IPA) of the changes observed in HD and HDL2 relative to control. A, the top canonical pathways enriched in the proteins that changed in the abundances in HD and HDL2, relative to control, sorted by the overlapping p-value; $\mathrm{B}$, the sorting of canonical pathways based on the activation $\mathrm{z}$-score $(\mathrm{z}$-score $>2$ or $<-2$ is considered significant); C, "Upstream analysis", designed to predict what regulators caused changes in protein expression and the directional state of regulator. 
S5
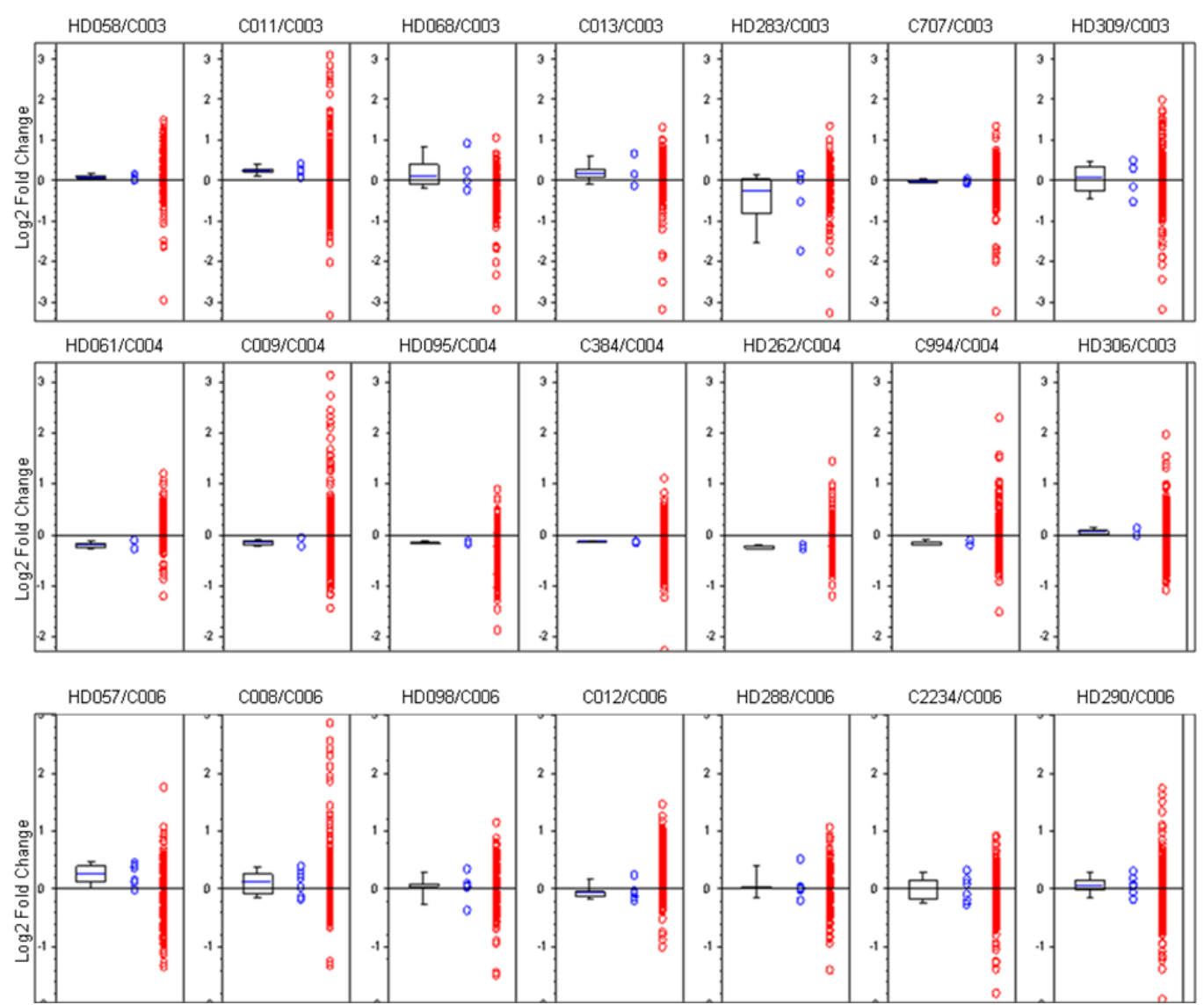

Figure S1 


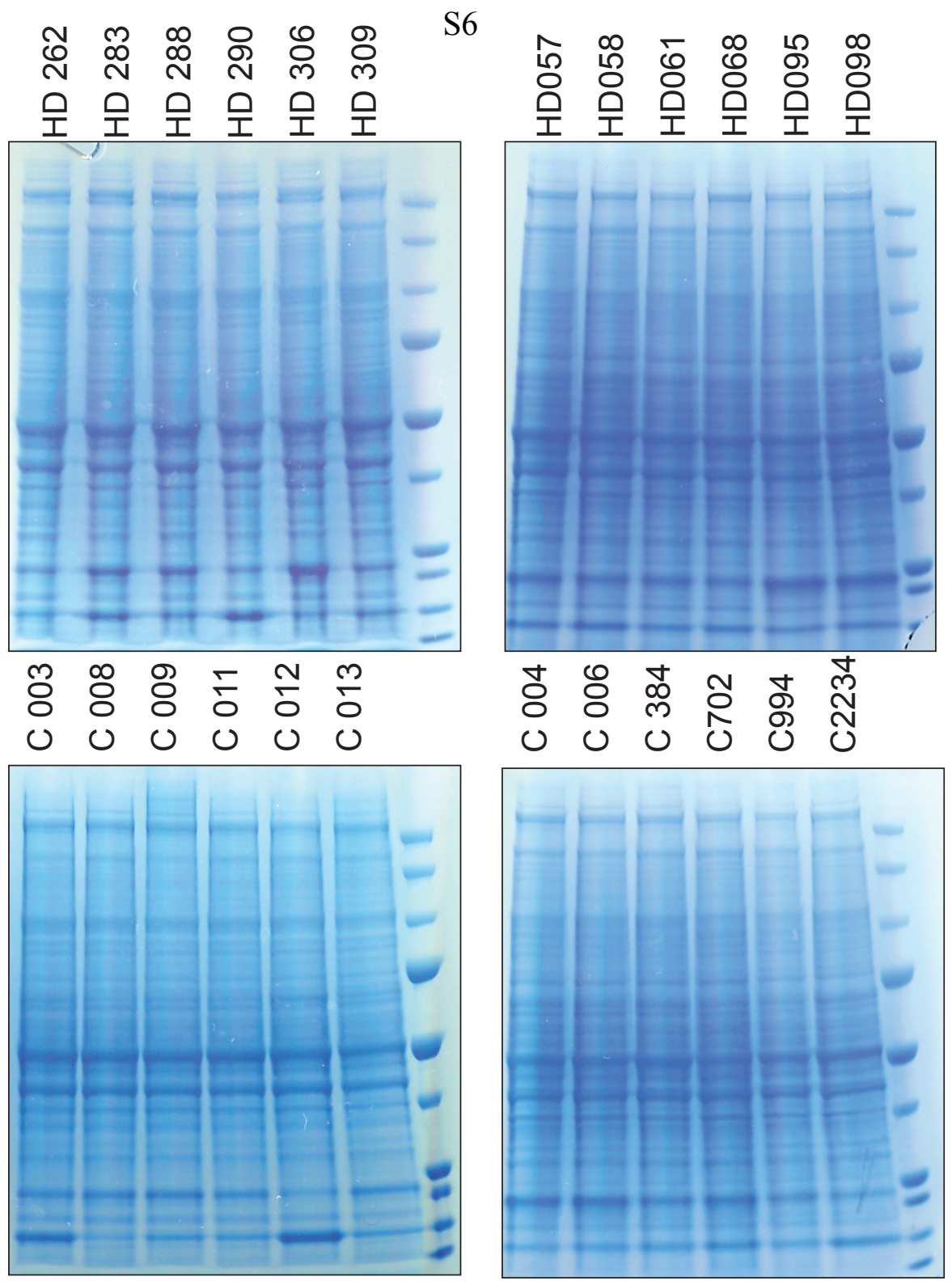

Figure S2 


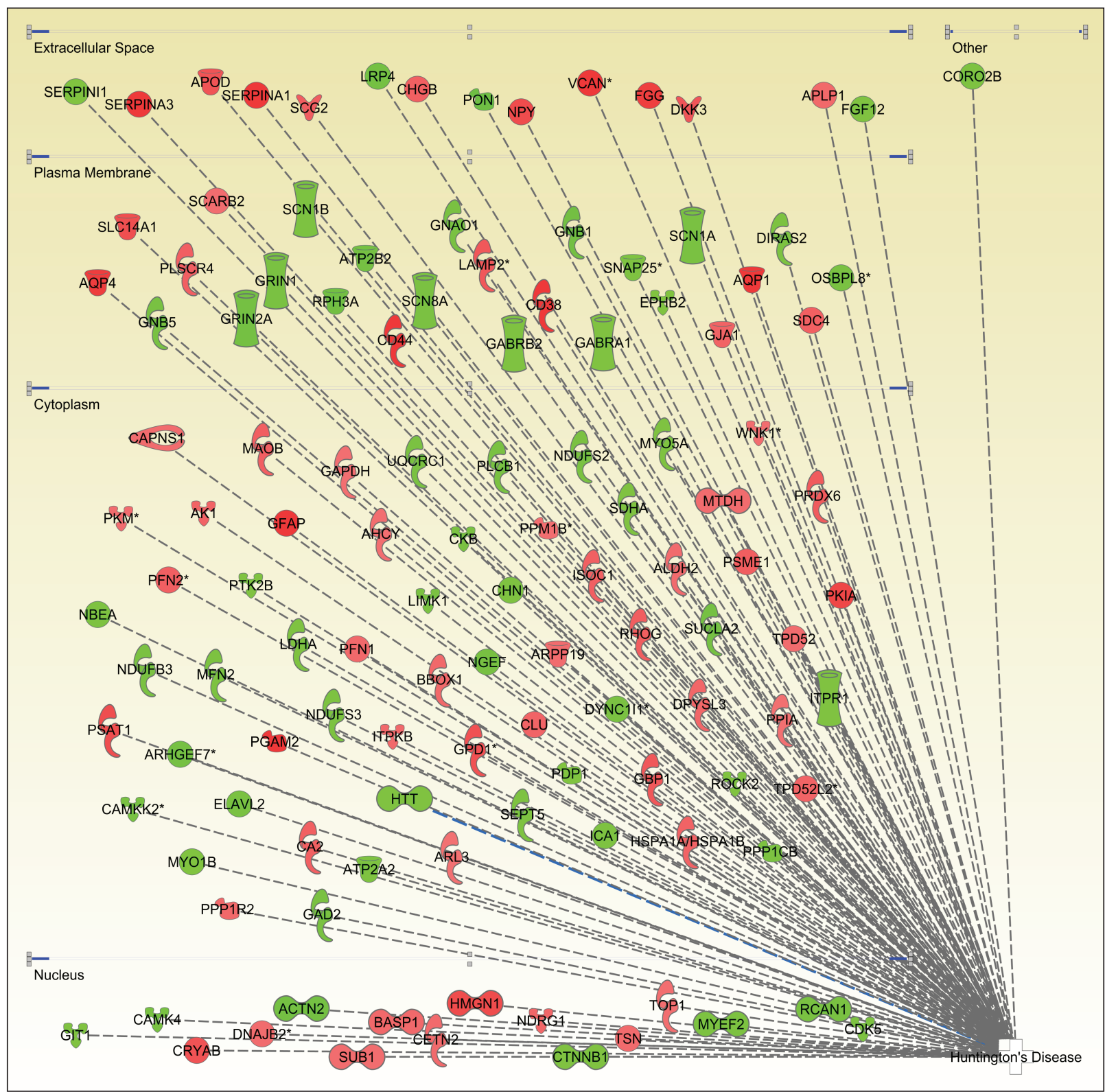

Figure S3 
W positive z-score $\square$ z-score $=0 \square$ negative z-score $\square$ no activity pattern available $\square$ - Ratio

Integrin Signaling

Protein Kinase A Signaling

Actin Cytoskeleton Signaling

Axonal Guidance Signaling

Signaling by Rho Family GTPases

Paxillin Signaling

Caveolar-mediated Endocytosis Signaling

14-3-3-mediated Signaling

RhoGDI Signaling

ERK/MAPK Signaling

Chemokine Signaling

RhoA Signaling

Agrin Interactions at Neuromuscular Junction

Germ Cell-Sertoli Cell Junction Signaling

Thrombin Signaling

p70s6K Signaling

Dopamine-DARPP32 Feedback in cAMP Signaling

Sertoli Cell-Sertoli Cell Junction Signaling

ILK Signaling

Gap Junction Signaling

FAK Signaling

GNRH Signaling

Phospholipase C Signaling

Ephrin Receptor Signaling

Xenobiotic Metabolism Signaling

G-Protein Coupled Receptor Signaling

Synaptic Long Term Potentiation

Regulation of Cellular Mechanics by Calpain Protease

IL-8 Signaling

Tryptophan Degradation X (Mammalian, via Tryptamine)

Epithelial Adherens Junction Signaling

Semaphorin Signaling in Neurons
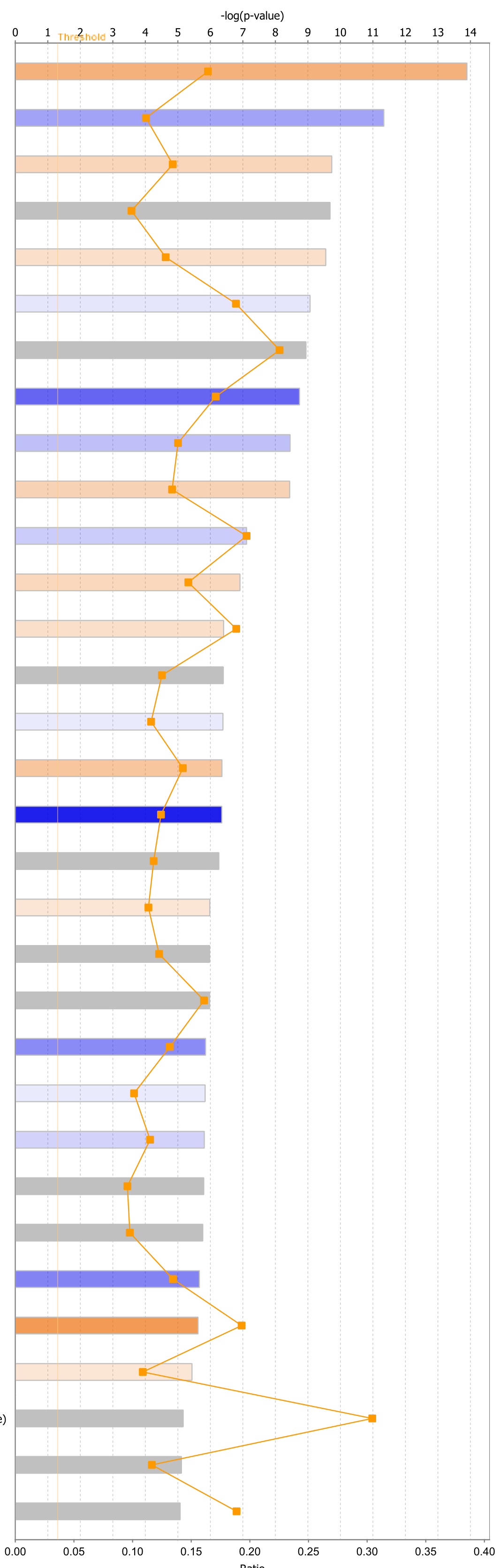

$\begin{array}{lllllllll}0.00 & 0.05 & 0.10 & 0.15 & \begin{array}{l}0.20 \\ \text { Ratio }\end{array} & 0.25 & 0.30 & 0.35 & 0.40\end{array}$

Figure S4 
A

$\begin{array}{r}\text { Canonical Pathway } \\ \text {-log(p-value) } \\ 0.00 E 000 \\ \hline \text { Signaling by Rho Family } 14.5\end{array}$

Signaling by Rho Family GTPases Integrin Signaling

Actin Cytoskeleton Signaling

RhoGDI Signaling

Protein Kinase A Signaling

RhoA Signaling

Axonal Guidance Signaling

Paxillin Signaling

14-3-3-mediated Signaling

Thrombin Signaling

ERK/MAPK Signaling

ILK Signaling

Ephrin Receptor Signaling

Germ Cell-Sertoli Cell Junction Signaling

Dopamine-DARPP32 Feedback in cAMP Sign...

Clathrin-mediated Endocytosis Signaling

Ephrin B Signaling

Melatonin Signaling

Semaphorin Signaling in Neurons

CREB Signaling in Neurons

Synaptic Long Term Potentiation

Huntington's Disease Signaling

Caveolar-mediated Endocytosis Signaling

Sertoli Cell-Sertoli Cell Junction Signaling

Cardiac $\beta$-adrenergic Signaling

p70S6K Signaling

FAK Signaling

Production of Nitric Oxide and Reactive Oxyg

Mitochondrial Dysfunction

Phospholipase C Signaling

Chemokine Signaling

IL-8 Signaling

CDK5 Signaling

Cardiac Hypertrophy Signaling

G $\alpha q$ Signaling

NRF2-mediated Oxidative Stress Response

Regulation of Cellular Mechanics by Calpain ..

Gap Junction Signaling

P2Y Purigenic Receptor Signaling Pathway

G-Protein Coupled Receptor Signaling

GNRH Signaling

PAK Signaling

tRNA Charging

Agrin Interactions at Neuromuscular Junction

Regulation of Actin-based Motility by Rho

Superpathway of Inositol Phosphate Compou.

Relaxin Signaling

Virus Entry via Endocytic Pathways

$\alpha$-Adrenergic Signaling

G Beta Gamma Signaling

Xenobiotic Metabolism Signaling

Pentose Phosphate Pathway

GABA Receptor Signaling

Epithelial Adherens Junction Signaling

Tryptophan Degradation X (Mammalian, via ...

Putrescine Degradation III

3-phosphoinositide Biosynthesis

Sphingosine-1-phosphate Signaling

Oxidative Phosphorylation
B

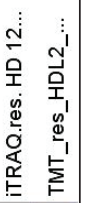

Activation z-score

Dopamine-DARPP32 Feedback in cAMP Sign...

Regulation of Cellular Mechanics by Calpain ..

CREB Signaling in Neurons

P2Y Purigenic Receptor Signaling Pathway

NRF2-mediated Oxidative Stress Response

GABA Receptor Signaling

Regulation of Actin-based Motility by Rho

Protein Kinase A Signaling

Gaq Signaling

Cardiac Hypertrophy Signaling

Phospholipase C Signaling

ERK/MAPK Signaling

Ephrin B Signaling

Integrin Signaling

p70S6K Signaling

Synaptic Long Term Potentiation

RhoA Signaling

14-3-3-mediated Signaling

Paxillin Signaling

RhoGDI Signaling

G Beta Gamma Signaling

GNRH Signaling

Relaxin Signaling

Agrin Interactions at Neuromuscular Junction

Production of Nitric Oxide and Reactive Oxyg.

IL-8 Signaling

CDK5 Signaling

Huntington's Disease Signaling

Actin Cytoskeleton Signaling

Sphingosine-1-phosphate Signaling

PAK Signaling

ILK Signaling

Signaling by Rho Family GTPases

Chemokine Signaling

Melatonin Signaling

Ephrin Receptor Signaling

$\alpha$-Adrenergic Signaling

Cardiac $\beta$-adrenergic Signaling

Thrombin Signaling

Virus Entry via Endocytic Pathways

Tryptophan Degradation X (Mammalian, via ...

Mitochondrial Dysfunction

tRNA Charging

Pentose Phosphate Pathway

Gap Junction Signaling

Semaphorin Signaling in Neurons

G-Protein Coupled Receptor Signaling

3-phosphoinositide Biosynthesis

Sertoli Cell-Sertoli Cell Junction Signaling

Clathrin-mediated Endocytosis Signaling

FAK Signaling

Caveolar-mediated Endocytosis Signaling

Superpathway of Inositol Phosphate Compou...

Putrescine Degradation III

Axonal Guidance Signaling

Oxidative Phosphorylation

Epithelial Adherens Junction Signaling

Xenobiotic Metabolism Signaling

Germ Cell-Sertoli Cell Junction Signaling
C

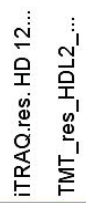

(1)

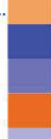

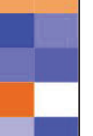

PPARGC1A

HNF4A

Stat3-Stat3

JUN

TP53

NFE2L2

HIF1A

SOX11

EGF

CREB1

SMARCA4

HGF

EPAS1

PDGF BB

IKZF1

PDX1

EGR1

AGT

SP1

Creb

GH1

SNAI1

NFkB (complex)

NFKB1

FOXA2

STAT3

BMP7

IRF7

TFAM

COPS5

CCND1

CTGF

ATF4

CBX5

GLI2

Nfat (family)

TRIM24 


\section{Supplementary tables legends}

\section{Table S1. List of proteins, present in at least one iTRAQ experiment for HD vs control}

dataset. 4789 proteins were identified and quantified based on more than one peptide with a confidence threshold 1\% False Discovery Rate based on a concatenated decoy database search. Description of the columns: first column- Protein Group Accession; log2FC- estimate of the $\log 2$-fold-change corresponding to the effect size; t.ord -ordinary t-statistic; t.mod- moderated tstatistic; p.ord -ordinary p-value corresponding to the ordinary t-statistic; p.mod -moderated pvalue corresponding to the moderated t-statistic; q.ord ordinary q-value corresponding to the ordinary t-statistic; q.mod- moderated q-value corresponding to the moderated t-statistic; df.r residual degrees of freedom associated with ordinary t-statistic and p-value; df.0 degrees of freedom associated with s2.0; s2.0- estimated prior value for the variance; s2 -sample variance; s2.-post posterior value for the variance.

Table S2. List of proteins, significantly changed in abundance in HD relative to control brain tissues, annotated by IPA for functional analysis. 1167 proteins were found significantly changed based on the pairwise comparison between $12 \mathrm{HD}$ and 12 control cases (frontal motor cortex) using iTRAQ labeling. Only proteins with $\mathrm{p}<0.05, \mathrm{q}<0.05$ are shown. The ratio values are converted to fold change where the negative inverse $(-1 / x)$ is taken for values between 0 and 1.

Table S3. List of proteins, present in at least one TMT experiment for HDL2 vs control dataset. 5567 proteins were identified and quantified based on more than one peptide with a confidence threshold 1\% False Discovery Rate based on a concatenated decoy database search. Description of the columns is same as in Table S1. 
Table S4. List of proteins, significantly changed in abundance in HDL2 relative to control brain tissues, annotated by IPA for functional analysis. 707 proteins were found significantly changed based on the pairwise comparison between 6 HDL2 and 6 control cases (frontal motor cortex) using TMT labeling. Only proteins with $\mathrm{p}<0.05, \mathrm{q}<0.05$ are shown. The ratio values are converted to fold change where the negative inverse $(-1 / \mathrm{x})$ is taken for values between 0 and 1 . Table S5. Comparison of our data to previously published data sets. Each row shows a protein significantly changed in abundance in HD relative to control brain in our study, and in at least one of the 3 gene profiling or 2 proteomics studies, or have been previously identified as $\mathrm{Htt}$ interactor in one out of $2 \mathrm{Htt}$ interaction studies. The following comparisons are shown: with gene profiling studies- Hodges et al, 2006 (Table 3) ${ }^{44}$; Langfelder et al, 2016 (Supplementary Table 10) ${ }^{55}$; Lin et al, 2016 (Table S2) ${ }^{56}$; with proteomics studies- Sorolla, et al, 2012 ${ }^{26}$, and Chen, et al, 201227; with interactions studies- Tourette et al, 2014 (primary Htt interactors from Supplemental Table 1) $)^{57}$ and Shirasaki,et al, 2012 (Table S6) ${ }^{21}$. Corresponding references for previous transcriptomics, proteomics and interactions reports are indicated in 3 corresponding reference columns. Proteins significantly up-regulated in our data set are shown in red, and down-regulated-in green. Genes/proteins that changed in the opposite direction in previously published reports are marked with asterisk in the corresponding reference column. Isoforms or different subunits found in previous studies are shown in blue in the reference columns. 
http://ingenuity.force.com/ipa/articles/Feature_Description/Legend

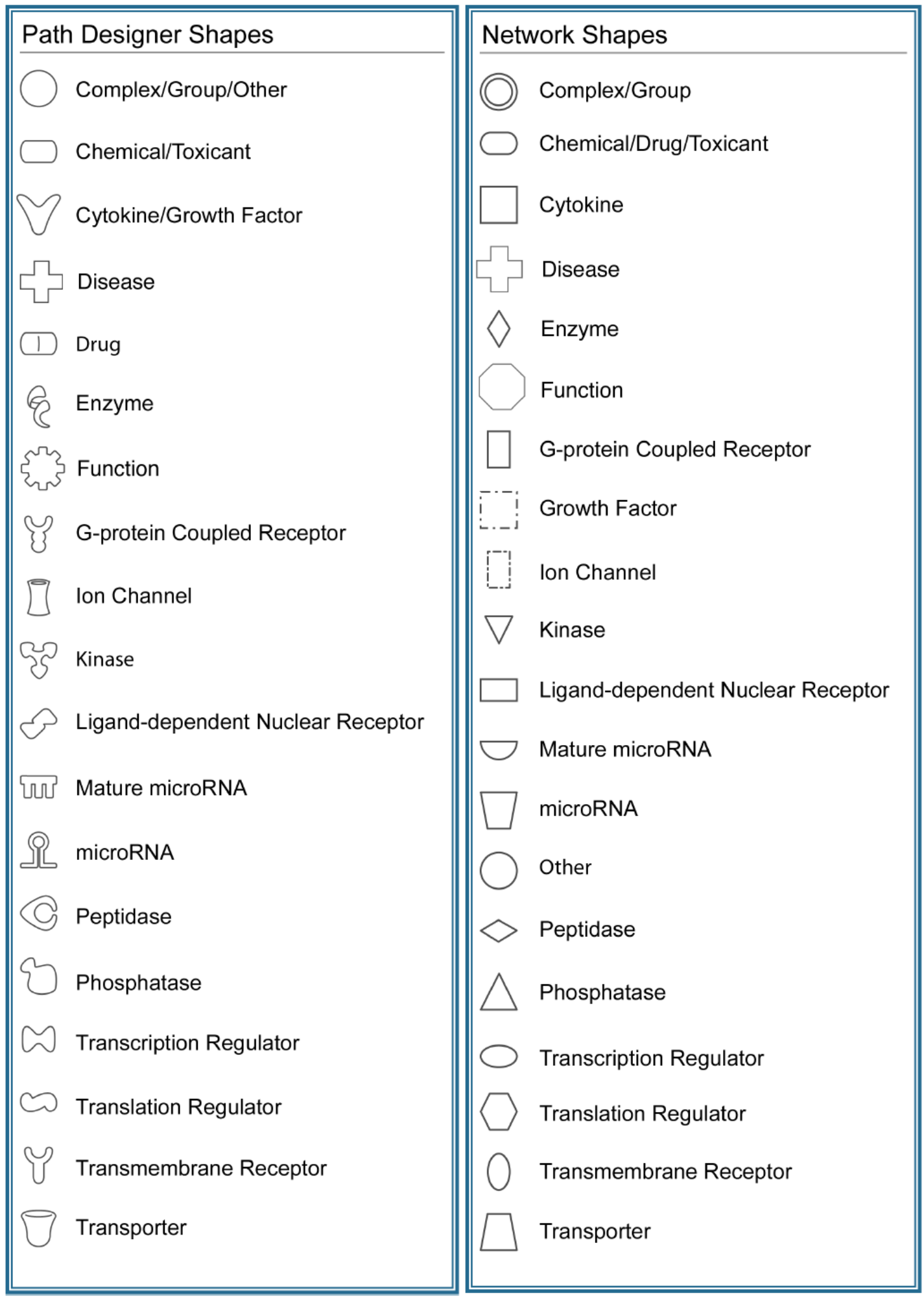

